\title{
INVASÃO POR ESPÉCIES ARBÓREAS EXÓTICAS EM REMANESCENTES FLORESTAIS NO PLANALTO SUL CATARINENSE ${ }^{1}$
}

André Luiz Guidini², Ana Carolina Silva ${ }^{3}$, Pedro Higuchi ${ }^{3}$, Angélica Dalla Rosa ${ }^{4}$, Fábio Rodrigues Spiazzi ${ }^{5}$, Marcelo Negrini ${ }^{6}$, Tiago de Souza Ferreira² ${ }^{2}$ Bruna Salami², Amanda Koche Marcon² e Fernando Buzzi Junio $^{4}$

\begin{abstract}
RESUMO - Este estudo objetivou avaliar a invasão por espécies arbóreas exóticas em dois fragmentos (F1 e F2) de Floresta Ombrófila Mista (FOM) em Lages, SC. Foram alocadas 25 parcelas por fragmento, distribuídas em cinco transeções de 20 x 100 m, perpendiculares às bordas e com distância de $100 \mathrm{~m}$ entre si, onde foram avaliados os indivíduos dos estratos adulto (DAP - diâmetro à altura do peito $\geq 5 \mathrm{~cm}$ ) e regenerante (DAP $<5 \mathrm{~cm}$ e altura $\geq 10 \mathrm{~cm}$ ). Esses foram identificados e mensurados (circunferência e altura). Foi calculado, para as espécies de cada estrato e de cada fragmento, o Índice de Invasão Biológica (IIB). A relação entre o IIB e a distância da borda foi verificada por meio de regressões lineares simples. O agrupamento de espécies exóticas e nativas foi determinado por meio de correlações de Spearman e de dendrogramas. Foram amostrados 3.701 indivíduos distribuídos em 105 espécies, sendo cinco espécies invasoras. No F1 houve valores de IIB relativamente baixos $(0,05$ e 0,54$)$, com Pinus taeda L., obtendo-se a maior participação na invasão, e esse esteve agrupado com espécies nativas pioneiras. Os IIB's no F2 foram altos $(0,61$ e 1,96$)$, principalmente pela elevada participação de Ligustrum lucidum W.T. Aiton., que ficou agrupado a espécies típicas da FOM. Não foi observada relação entre a distância da borda e a intensidade da invasão biológica. Os resultados indicaram que os fragmentos apresentaram diferentes padrões de invasão biológica, determinados pela natureza da matriz de entorno e pelas características ecológicas das espécies invasoras.
\end{abstract}

Palavras-chave: Índice de Invasão Biológica; Floresta Ombrófila Mista; Floresta com Araucária.

\section{INVASION BY EXOTIC TREE SPECIES IN FOREST REMNANTS IN “PLANALTO SUL CATARINENSE" REGION}

\begin{abstract}
This study evaluated the invasion by exotic tree species in two Mixed Ombrophilous Forest (FOM) fragments (F1 and F2) in Lages, SC. A total of 25 plots were allocated in each fragment, distributed in five transects, $20 \times 100 \mathrm{~m}$, perpendicular to the edges and, at least, $100 \mathrm{~m}$ apart from each other, where the adult (dbh-diameter at breast height $\geq 5 \mathrm{~cm}$ ) and the regenerative ( $\mathrm{dbh}<5 \mathrm{~cm}$ and height $\geq 10 \mathrm{~cm}$ ) component individuals were evaluated. These were identified and measured (circumference and height). For each component and fragment, the biological invasion index (BII) was determined. The relation between BII and the distance from the edge was evaluated by simple linear regressions. The grouping among exotic and native species was analyzed through Spearman correlation and clusters. A total of 3,701 individuals
\end{abstract}

\footnotetext{
${ }^{1}$ Recebido em 21.10.2013 aceito para publicação em 29.04.2014.

${ }^{2}$ Mestrado em Engenharia Florestal, Universidade do Estado de Santa Catarina, SC, Brasil. E-mail: <andrelg85@yahoo.com.br>, <tiagoferreira@florestal.eng.br>, <brunaflorestal@yahoo.com.br> e <amandamarcon@yahoo.com.br>.

${ }^{3}$ Departamento de Engenharia Florestal, Universidade do Estado de Santa Catarina, SC, Brasil. E-mail: <carol_sil4@yahoo.com.br> e $<$ higuchip@gmail.com>.

${ }^{4}$ Graduando em Engenharia Florestal, Universidade do Estado de Santa Catarina, SC, Brasil. E-mail: <angelica.dalla.rosa@hotmail.com> e <buzzifjr@hotmail.com>.

${ }^{5}$ Mestrado em Manejo do Solo, Universidade do Estado de Santa Catarina, SC, Brasil. E-mail: <maxplus2007@yahoo.com.br>.

${ }^{6}$ Programa de Pós-Graduação em Recursos Naturais, Universidade Federal de Roraima, RR, Brasil. E-mail: <engfmarcelonegrini@hotmail.com>.
} 
distributed in 105 species were sampled, being five invasive species. In F1 a relative low value of BII (0.05 and 0.54) occurred, with Pinus taeda $L$. as the most expressive species in the invasion, and it was grouped with native pioneers species. The BII's in F2 were elevated (0.61 and 1.96), mainly due to the expressive participation of Ligustrum lucidum W.T. Aiton., grouped with typical species of FOM. No relation between the distance from the edges and the intensity of the invasion were observed. The results demonstrated that the fragments showed different biological invasion patterns that were determined by the nature of surrounding matrix and the ecological characteristics of the invasive species.

Keywords: Biological Invasion Index; Mixed Ombrophylous Forest; Araucaria Forest.

\section{INTRODUÇÃO}

A invasão biológica acontece, em geral, pela introdução de espécies pelo homem em ambientes onde elas não ocorrem naturalmente. As espécies introduzidas, por não terem coevoluído com as espécies nativas, muitas vezes não possuem predador natural, o que pode fazer que espécies exóticas tenham vantagem competitiva para se estabelecerem e se desenvolverem (VALÉRY et al., 2008). Segundo Barbieri e Stumpt (2008), menos de $10 \%$ das espécies introduzidas conseguem se adaptar ao novo ambiente e menos e $10 \%$ delas se tornam invasoras. E estima-se que entre 0,1\% e 1\% das espécies introduzidas se tornam, de fato, invasoras. No entanto, mesmo com esse baixo percentual, a invasão biológica representa uma das principais causas da perda de diversidade no mundo (HELENO et al., 2010).

$\mathrm{Na}$ floresta, as espécies invasoras afetam os processos ecológicos dos ecossistemas, como a ciclagem de nutrientes, a produtividade vegetal, as cadeias tróficas, a polinização, a dispersão de sementes e a sucessão ecológica, além de interferir na densidade de espécies nativas, na fisionomia e nas taxas de decomposição (ZILLER, 2000), ocasionando danos ambientais e prejuízos econômicos. No Brasil, diversas espécies invasoras têm causado problemas, como as citadas pelo Instituto Horus (2013), destacando-se como invasoras de fragmentos florestais no Planalto SulCatarinense espécies dos gêneros Pinus, que são amplamente cultivadas na região para fins madeireiros; e Ligustrum, que são cultivadas no ambiente urbano para arborização das cidades.

Considerando que fragmentos florestais são influenciados pelos componentes existentes na matriz circundante (LAURANCE et al., 2002), a participação de espécies invasoras ao longo de gradientes de borda pode refletir a sua autoecologia. Nesse sentido, são necessários estudos que quantifiquem o número de indivíduos invasores em fragmentos florestais naturais e sua abundância nas bordas dos fragmentos, objetivando conhecer o grau de invasão e, assim, indicar a necessidade de manejo dos fragmentos, a fim de diminuir essas espécies invasoras e mitigar seus efeitos deletérios. Assim, os objetivos deste trabalho foram: i) quantificar a participação de espécies arbóreas invasoras nos estratos adulto e regenerante de dois fragmentos florestais localizados no Planalto Sul-Catarinense; ii) verificar se existe variação na intensidade de invasão em razão da distância da borda desses fragmentos; e iii) verificar a existência de agrupamentos entre espécies arbóreas exóticas e nativas nesses locais.

\section{MATERIAL E MÉTODOS}

Foram estudados dois remanescentes florestais em avançado estágio de sucessão localizados em Lages, Planalto Sul-Catarinense, cuja vegetação pode ser classificada como Floresta Ombrófila Mista Montana, segundo o IBGE (2012). Ambos estão próximos à área urbana e a plantios de Pinus spp. Um dos remanescentes denominado F1 e que está inserido no Parque Natural Municipal de Lages (PARNAMUL) fazia divisa com um plantio comercial de Pinus spp., separado apenas por um aceiro. O outro fragmento (F2) era um remanescente pertencente à área da Empresa de Pesquisa Agropecuária e Extensão Rural de Santa Catarina (EPAGRI) e estava inserido na área urbana de Lages, cuja arborização é composta, predominantemente, por Ligustrum lucidum W.T. Aiton. Lages está localizada na latitude de $27^{\circ} 48^{\prime} 58$ ' 'Sul e longitude de 50¹9’30”, Oeste, com altitude em torno de $916 \mathrm{~m}$. O clima predominante na região é $\mathrm{Cfb}$, de acordo com a classificação de Köppen, sendo a precipitação anual média de 1.479,48 mm, com chuvas bem distribuídas durante o ano, e temperatura anual média de $16^{\circ} \mathrm{C}$. O município está inserido na Bacia Hidrográfica do Rio Canoas e do Rio Pelotas, com topografia, em sua maior parte, suave-ondulada a ondulada. 
Em cada fragmento, foram alocadas cinco transeções de $20 \mathrm{~m}$ de largura e $100 \mathrm{~m}$ de comprimento, perpendiculares à borda da floresta, com distância mínima de 100 m entre si. Cada transecção foi dividida em cinco parcelas de 20 × 20 m, totalizando 25 parcelas de 400 $\mathrm{m}^{2}$ por fragmento. Essas parcelas possuíam diferentes distâncias da borda, e cada fragmento tinha cinco parcelas em contato com a borda e cinco em cada uma das distâncias da borda: 20 m, 40 m, 60 m e 80 m. Os indivíduos arbóreos vivos que apresentaram diâmetro à altura do peito (DAP) medido a $1,30 \mathrm{~m}$ do solo, $\geq 5,0 \mathrm{~cm}$, foram amostrados nessas parcelas e considerados adultos. Estes foram identificados com plaquetas, sendo registrada a identificação botânica da espécie (classificados segundo o sistema APG III, 2009) e mensurados seus DAPs. Para avaliação do estrato regenerante (indivíduos pertencentes a espécies arbóreas com DAP $<5 \mathrm{~cm}$ e altura $\leq 10 \mathrm{~cm}$ ), cada parcela foi subdivida em subparcelas com área variável de acordo com a classe de tamanho da planta, seguindo-se metodologia adaptada de Volpato (1994): i) Classe 1, plantas com altura entre $10 \mathrm{~cm}$ e $1 \mathrm{~m}$, avaliadas em $5 \mathrm{~m}^{2}$; ii) Classe 2, plantas com altura entre 1 e $3 \mathrm{~m}$, avaliadas em $10 \mathrm{~m}^{2}$; e iii) Classe 3, plantas com altura $>$ que $3 \mathrm{~m}$ e DAP $<$ que $5 \mathrm{~cm}$, avaliadas em $20 \mathrm{~m}^{2}$. Os indivíduos nas subparcelas foram identificados e tiveram sua classe de altura registrada.

A comparação da riqueza entre os estratos adulto e regenerante nos dois fragmentos foi realizada por meio da técnica de rarefação, com 1.000 permutações, padronizando a amostragem em função do número de indivíduos na regeneração natural. A suficiência amostral para determinar a riqueza de espécies inventariadas em cada fragmento e em cada estrato (adulto e regenerante) foi determinada pela análise da curva de acumulação de espécies, construída pelo método de aleatorização, com 1.000 permutações. A estrutura da comunidade arbórea adulta foi descrita a partir das estimativas para cada espécie e dos Índices de Valor de Importância (IVI), calculados segundo MuellerDombois e Ellemberg (1974) pela média das densidades, frequências e dominâncias relativas. A estrutura da comunidade arbórea regenerante foi estimada pelos Índices de Regeneração Natural (IRN) das espécies, calculados por meio das médias dos valores de frequência e densidade relativa.

Para quantificar o nível de invasão biológica no estrato adulto, foi proposto um Índice de Invasão Biológica (IIB): IVI/IVI $_{\max }$, em que $\Sigma$ IVI é o somatório do(s) valor(es) do(s) IVI(s) da(s) espécie(s) exótica(s); e $I V I_{\max }$ é o IVI da espécie nativa de maior valor encontrado. Se IIB $\geq 1$, o grupo das espécies invasoras possui a maior importância relativa na comunidade; se $1>$ IIB $\geq 0,5$, as invasoras possuem importância relativa inferior à da espécie nativa de maior IVI, porém com valor superior à metade do IVI da espécie nativa mais importante; e se IIB $<0,5$, o grupo das invasoras possui importância relativa inferior à metade do valor de IVI da espécie nativa de maior valor de importância. Para quantificar o nível de invasão biológica no estrato regenerativo, foi utilizado o Índice de Invasão Biológica, calculado de forma similar: $\Sigma$ IRN/IRN ${ }_{\text {max }}$, em que $\Sigma$ IRN é o somatório do(s) valor(es) do(s) IRN(s) da(s) espécie(s) exótica(s); e $I R N_{\max }$ é o IRN da espécie nativa de maior valor encontrado. A interpretação do valor do índice foi semelhante ao do componente adulto, considerando, em vez de IVI, o IRN. A relação do IIB com a distância da borda foi avaliada por meio de regressões lineares simples. Para verificar a existência de agrupamentos entre espécies exóticas invasoras e nativas, foram utilizadas matrizes de abundância das espécies ( $\geq$ cinco indivíduos) por parcelas para cada fragmento e estrato. Para reduzir a discrepância entre a representatividade das espécies mais e menos abundantes, as matrizes passaram por uma transformação de Hellinger (BOCARD et al., 2011). Posteriormente, foram determinadas as correlações de Spearman entre as espécies, que foram utilizadas para a construção de dendrogramas, por meio do algoritmo de Ward (LEGENDRE; BLANCHER, 2008). A qualidade dos dendrogramas foi verificada por meio dos coeficientes de correlações cofenéticas (OKSANEN, 2012). As análises foram realizadas em planilhas eletrônicas e pelo programa R (R DEVELOPMENT CORE TEAM, 2013), junto com o pacote Vegan (OKSANEN et al., 2013).

\section{RESULTADOS}

Nos dois fragmentos foram amostrados 2.763 indivíduos adultos e 938 regenerantes, distribuídos em 105 espécies e 42 famílias (Tabela 1). No estrato adulto do F2, 12 indivíduos não puderam ser identificados por estarem sem folhas. Comparando a riqueza nos fragmentos e estratos, apenas no F1 verificou-se diferença entre a riqueza do componente adulto (55,3 $\pm 2,7$ espécies) e regenerante (44 espécies). Em todas as áreas e estratos, com a inclusão da última parcela, o aumento da riqueza foi inferior a $2 \%$, e as curvas tenderam à estabilidade, indicando que a amostragem foi adequada para quantificar a riqueza nas áreas. 
Tabela 1 - Espécies amostradas nos fragmentos e estratos estudados em Lages, SC, sendo: AF1 = estrato adulto do fragmento no PARNAMUL, RF1 = estrato regenerante do fragmento no PARNAMUL, AF2 = estrato adulto do fragmento na EPAGRI e RF2 = estrato regenerante do fragmento na EPAGRI.

Table 1 - Sampled species in fragments and components in Lages, SC, being: AF1 = adult component of PARNAMUL fragment, $R F 1$ = regenerative component of PARNAMUL fragment, AF2 = adult component of EPAGRI fragment and $R F 2$ = regenerative component of EPAGRI fragment.

\begin{tabular}{|c|c|c|c|c|c|c|}
\hline Famílias & Espécies & AF1 & RF1 & AF2 & RF2 & Total \\
\hline \multirow[t]{2}{*}{ Anacardiaceae } & Lithraea brasiliensis Marchand & 37 & - & 73 & 2 & 112 \\
\hline & Schinus terebinthifolius Raddi & 4 & - & 5 & - & 9 \\
\hline Annonaceae & $\begin{array}{c}\text { Annona rugulosa (Schltdl.) } \\
\text { H.Rainer }\end{array}$ & 5 & 9 & 3 & - & 17 \\
\hline \multirow[t]{4}{*}{ Aquifoliaceae } & Ilex dumosa Reissek & 1 & - & 6 & - & 7 \\
\hline & Ilex microdonta Reissek & 1 & - & 6 & - & 7 \\
\hline & Ilex paraguariensis A.St.-Hil. & 1 & - & 2 & 2 & 5 \\
\hline & Ilex theezans Mart. ex Reissek & 13 & 1 & 115 & 13 & 142 \\
\hline Araliaceae & Oreopanax fulvus Marchal & 1 & 1 & 1 & - & 3 \\
\hline Araucariaceae & $\begin{array}{c}\text { Araucaria angustifolia (Bert.) } \\
\text { Kuntze }\end{array}$ & 26 & 5 & 7 & - & 38 \\
\hline \multirow[t]{6}{*}{ Asteraceae } & $\begin{array}{c}\text { Baccharis dentata (Vell.) } \\
\text { G.Barroso }\end{array}$ & - & - & - & 1 & 1 \\
\hline & $\begin{array}{c}\text { Dasyphyllum spinescens (Less.) } \\
\text { Cabrera }\end{array}$ & - & 3 & 2 & 1 & 6 \\
\hline & $\begin{array}{c}\text { Dasyphyllum tomentosum (Spreng.) } \\
\text { Cabrera }\end{array}$ & 10 & 1 & 3 & 3 & 17 \\
\hline & $\begin{array}{c}\text { Gochnatia polymorpha (Less.) } \\
\text { Cabrera }\end{array}$ & 10 & - & 64 & 1 & 75 \\
\hline & Piptocarpha angustifolia Dusén & 1 & - & 1 & - & 2 \\
\hline & $\begin{array}{c}\text { Vernonanthura discolor (Spreng.) } \\
\text { H.Rob. }\end{array}$ & 10 & - & 29 & - & 39 \\
\hline Berberidaceae & Berberis laurina Billb. & - & - & - & 2 & 2 \\
\hline \multirow[t]{2}{*}{ Bignoniaceae } & $\begin{array}{l}\text { Handroanthus albus } \\
\text { (Cham.) Mattos }\end{array}$ & - & - & 1 & - & 1 \\
\hline & Jacaranda puberula Cham. & 8 & 2 & 56 & 17 & 83 \\
\hline Canellaceae & Cinnamodendron dinisii Schwacke & 32 & 2 & 88 & 9 & 131 \\
\hline Cannabaceae & Celtis brasiliensis (Gardner) Planch. & - & 5 & - & - & 5 \\
\hline \multirow[t]{2}{*}{ Cardiopteridaceae } & $\begin{array}{c}\text { Citronella gongonha (Mart.) } \\
\text { R.A.Howard }\end{array}$ & - & - & 1 & - & 1 \\
\hline & Citronella paniculata (Mart.) Howard & 1 & 4 & - & - & 5 \\
\hline Celastraceae & Maytenus dasyclada Mart. & 4 & - & 19 & 40 & 63 \\
\hline Clethraceae & Clethra scabra Pers. & 9 & 3 & 39 & - & 51 \\
\hline Cunoniaceae & Lamanonia ternata Vell. & 1 & - & - & - & 1 \\
\hline Cyatheaceae & Cyatheaceae sp.1 & - & - & 8 & - & 8 \\
\hline Dicksoniaceae & Dicksonia sellowiana Hook. & 10 & - & 38 & 17 & 65 \\
\hline Elaeocarpaceae & Sloanea monosperma Vell. & 1 & - & - & - & 1 \\
\hline \multirow[t]{2}{*}{ Erythroxylaceae } & $\begin{array}{l}\text { Erythroxylum cuneifolium } \\
\text { (Mart.) O.E.Schulz }\end{array}$ & - & - & 4 & - & 4 \\
\hline & Erythroxylum deciduum A.St.-Hil. & 15 & - & 6 & 2 & 23 \\
\hline Escalloniaceae & Escallonia bifida Link \& Otto & - & - & 3 & 1 & 4 \\
\hline \multirow[t]{3}{*}{ Euphorbiaceae } & Sapium glandulosum (L.) Morong & 3 & - & 2 & - & 5 \\
\hline & Sebastiania brasiliensis Spreng. & 7 & 1 & - & 1 & 9 \\
\hline & $\begin{array}{c}\text { Sebastiania commersoniana (Baill.) } \\
\text { L.B.Sm. \& Downs }\end{array}$ & 21 & - & 161 & 36 & 218 \\
\hline \multirow[t]{5}{*}{ Fabaceae } & Dalbergia frutescens (Vell.) Britton & 4 & 25 & 19 & 20 & 68 \\
\hline & Inga lentiscifolia Benth. & 3 & - & - & - & 3 \\
\hline & Inga sp. & - & 2 & - & - & 2 \\
\hline & Machaerium sp. & - & - & - & 2 & 2 \\
\hline & Mimosa scabrella Benth. & 2 & - & 9 & - & 11 \\
\hline
\end{tabular}

Revista Árvore, Viçosa-MG, v.38, n.3, p.469-478, 2014 
Tabela 1 - Cont.

Table 1 - Cont.

Lauraceae

Mimosoideae sp.

Cinnamomum amoenum (Nees

\& Mart.) Kosterm.

$\begin{array}{lllllll}\text { Nectandra megapotamica (Spreng.) Mez } & 15 & 12 & 2 & 1 & 30\end{array}$

$\begin{array}{llllll}\text { Ocotea puberula (Rich.) Nees } & 8 & 1 & 4 & 1 & 14\end{array}$

$\begin{array}{lllllll}\text { Ocotea pulchella (Nees \& Mart.) Mez } & 9 & 4 & 29 & 9 & 51\end{array}$

Lythraceae

Lafoensia vandelliana

Cham. \& Schltdl.

Melastomataceae

Miconia cinerascens Miq.

Miconia ramboi Brade

Meliaceae

Tibouchina cf. sellowiana (Cham.) Cogn.

Cabralea canjerana (Vell.) Mart.

Myrtaceae

Acca sellowiana

(O.Berg) Burret

Blepharocalyx salicifolius (Kunth) O.Berg

Calyptranthes concinna DC.

Campomanesia xanthocarpa O.Berg

Eugenia hiemalis Cambess.

Eugenia pluriflora DC.

Eugenia pyriformis Cambess.

Eugenia uniflora L.

Eugenia uruguayensis Cambess.

Myrceugenia euosma (O.Berg) D.Legrand

Myrceugenia oxysepala (Burret)

D.Legrand \& Kausel Myrceugenia sp.

Myrcia guianensis (Aubl.) DC.

Myrcia hartwegiana (O.Berg) Kiaersk.

Myrcia hatschbachii D.Legrand

Myrcia laruotteana Cambess.

Myrcia palustris DC.

Myrcia splendens (Sw.) DC.

Myrcianthes gigantea (D.Legrand) D.Legrand

Myrciaria delicatula (DC.) O.Berg

Myrrhinium atropurpureum Schott

Siphoneugena reitzii D.Legrand N.I.

Oleaceae

Ligustrum lucidum W.T. Aiton.

Ligustrum sinense Lour.

Pinus elliottii Engel.

Pinus taeda $\mathrm{L}$.

Podocarpaceae

Podocarpus lambertii Klotzsch ex Endl.

Primulaceae

Myrsine coriacea (Sw.) R.Br. ex Roem. \& Schult.

Myrsine lorentziana (Mez) Arechav.

Myrsine umbellata Mart.

Proteaceae

Roupala montana Aubl.

Quillajaceae

Quillaja brasiliensis (A.St.-Hil. \& Tul.) Mart.

Rhamnaceae

Rhamnus sphaerosperma Sw.

Scutia buxifolia Reissek

\begin{tabular}{|c|c|c|c|c|}
\hline- & - & - & 1 & 1 \\
\hline 1 & 1 & - & - & 2 \\
\hline 15 & 12 & 2 & 1 & 30 \\
\hline 8 & 1 & 4 & 1 & 14 \\
\hline 9 & 4 & 29 & 9 & 51 \\
\hline - & - & - & 1 & 1 \\
\hline- & - & 1 & 2 & 3 \\
\hline - & - & 1 & - & 1 \\
\hline - & - & 1 & 3 & 4 \\
\hline 1 & - & - & - & 1 \\
\hline - & - & 1 & - & 1 \\
\hline 4 & 3 & 2 & - & 9 \\
\hline 79 & 2 & 9 & 6 & 96 \\
\hline 8 & 1 & 1 & - & 10 \\
\hline 1 & - & - & - & 1 \\
\hline 5 & 1 & 5 & 1 & 12 \\
\hline 21 & 2 & 5 & - & 28 \\
\hline 10 & 6 & - & - & 16 \\
\hline 11 & 2 & - & 1 & 14 \\
\hline 1 & - & 73 & 4 & 78 \\
\hline- & - & - & 1 & 1 \\
\hline 1 & - & - & - & 1 \\
\hline 12 & - & 23 & 3 & 38 \\
\hline - & - & - & 3 & 3 \\
\hline 72 & 25 & - & - & 97 \\
\hline 9 & 3 & 116 & 8 & 136 \\
\hline 25 & - & 49 & 29 & 103 \\
\hline 71 & 21 & 60 & 45 & 197 \\
\hline 1 & - & - & 1 & 2 \\
\hline 3 & - & 14 & 20 & 37 \\
\hline 5 & - & - & 3 & 8 \\
\hline - & - & 2 & - & 2 \\
\hline - & - & 12 & - & 12 \\
\hline 1 & - & 72 & 74 & 147 \\
\hline - & - & - & 11 & 11 \\
\hline - & - & 1 & - & 1 \\
\hline 6 & 1 & - & - & 7 \\
\hline - & - & 50 & 8 & 58 \\
\hline 11 & 6 & 31 & 8 & 56 \\
\hline 6 & 7 & 22 & 22 & 57 \\
\hline 8 & 9 & 54 & 4 & 75 \\
\hline 6 & 8 & - & - & 14 \\
\hline- & - & 3 & - & 3 \\
\hline- & - & 3 & - & 3 \\
\hline 3 & - & - & 1 & 4 \\
\hline
\end{tabular}


Tabela 1 - Cont.

Table 1 - Cont.

\begin{tabular}{|c|c|c|c|c|c|c|}
\hline Rosaceae & Prunus myrtifolia (L.) Urb. & 14 & 3 & 11 & 3 & 31 \\
\hline \multirow[t]{3}{*}{ Rutaceae } & Citrus limon (L.) Burm & - & - & 1 & - & 1 \\
\hline & $\begin{array}{c}\text { Zanthoxylum kleinii (R.S.Cowan) } \\
\text { P.G.Waterman }\end{array}$ & - & - & 46 & 4 & 50 \\
\hline & Zanthoxylum rhoifolium Lam. & 10 & 1 & 17 & 5 & 33 \\
\hline \multirow[t]{5}{*}{ Salicaceae } & Banara tomentosa Clos & 44 & 45 & - & 61 & 150 \\
\hline & Casearia decandra Jacq. & 159 & 10 & 191 & 11 & 371 \\
\hline & Casearia obliqua Spreng. & 21 & - & 15 & - & 36 \\
\hline & Xylosma ciliatifolia (Clos) Eichler & 4 & - & 5 & 3 & 12 \\
\hline & Xylosma tweediana (Clos) Eichler & - & - & 2 & 1 & 3 \\
\hline \multirow[t]{4}{*}{ Sapindaceae } & $\begin{array}{l}\text { Allophylus edulis (A.St.-Hil., } \\
\text { Cambess. \& A.Juss.) Radlk. }\end{array}$ & 10 & 12 & 6 & 16 & 44 \\
\hline & $\begin{array}{l}\text { Allophylus guaraniticus } \\
\text { (A.St.-Hil.) Radlk. }\end{array}$ & 5 & 7 & - & 7 & 19 \\
\hline & Cupania vernalis Cambess. & 32 & 30 & - & 1 & 63 \\
\hline & Matayba elaeagnoides Radlk. & 22 & 15 & 23 & 42 & 102 \\
\hline \multirow[t]{5}{*}{ Solanaceae } & Brunfelsia cuneifolia J.A.Schmidt & - & - & - & 3 & 3 \\
\hline & Cestrum intermedium Sendtn. & 1 & - & - & - & 1 \\
\hline & Solanum mauritianum Scop. & 1 & - & 1 & - & 2 \\
\hline & Solanum pseudoquina A.St.-Hil. & - & - & 1 & - & 1 \\
\hline & Solanum variabile Mart. & 1 & - & - & - & 1 \\
\hline Styracaceae & Styrax leprosus Hook. \& Arn. & - & 2 & 4 & 3 & 9 \\
\hline Symplocaceae & Symplocos uniflora (Pohl) Benth. & 1 & - & 24 & 1 & 26 \\
\hline Thymelaeaceae & Daphnopsis racemosa Griseb. & - & 1 & - & 22 & 23 \\
\hline \multirow[t]{4}{*}{ Winteraceae } & Drimys brasiliensis Miers & 5 & 1 & 14 & 8 & 28 \\
\hline & Total de indivíduos & 985 & 306 & 1778 & 632 & 3701 \\
\hline & Total de espécies & 73 & 44 & 71 & 61 & 105 \\
\hline & $\begin{array}{c}\text { Total de espécies padronizadas } \\
\text { pela rarefação }\end{array}$ & $\begin{array}{c}55,3 \\
( \pm 2,7)\end{array}$ & 44 & $\begin{array}{c}58,0 \\
( \pm 2,6)\end{array}$ & 61 & \\
\hline
\end{tabular}

Myrtaceae foi a família que apresentou o maior número de espécies (22) em ambos os componentes. Em nível específico, Casearia decandra Jacq. destacou-se com a maior abundância (371 indivíduos), principalmente no estrato adulto, pois foi a espécie que apresentou o maior IVI nesse estrato em ambos os fragmentos (IVI-F1 = 9,55\%; IVI-F2 =6,16\%). No estrato regenerante, as espécies de maior IRN foram outras: no F1 foi Banara tomentosa Closs, com 12,12\%; e no F2, Ligustrum lucidum, com 13,39\%.

Dos indivíduos amostrados (3.701), 167 (4,5\%) eram invasores, pertencentes a cinco espécies $(4,8 \%)$ : Ligustrum lucidum (147); Ligustrum sinense Lour (11); Pinus taeda L. (sete), Pinus elliottii Engel (um) e Citrus limon (L.) Burm (um). A participação de Ligustrum lucidum entre as espécies de maior importância no F2 (primeira dos regenerantes e oitava dos adultos) refletiu em altos valores de IIB (Tabela 2). O IIB no estrato adulto desse fragmento foi de 0,61 , superior à metade do IVI da espécie nativa mais importante, enquanto no estrato regenerante foi de 1,95, cujo grupo de invasoras possui a maior importância na comunidade. No F1, o IIB foi relativamente baixo para o estrato adulto $(0,05)$ e no regenerante $(0,54)$, superior à metade do IRN da espécie nativa mais importante.

Não foram observadas relações lineares significativas entre IIB e distância da borda, considerando um gradiente de 0 a $100 \mathrm{~m}$. Enquanto no F1 houve invasão na borda (0 m), a $60 \mathrm{~m}$ e a $80 \mathrm{~m}$, no F2 foi observada invasão biológica ao longo de todo o gradiente.

Por meio dos dendrogramas produzidos (correlação cofenética $p<0,001$ ), foi possível identificar o compartilhamento de hábitats das espécies exóticas invasoras com as espécies nativas. No componente adulto do F1 (Figura 1A), os indivíduos de Pinus taeda (PI.TAE) ocorreram agrupados a Vernonanthura discolor (Spreng.) H.Rob. (VE.DIS), Myrsine umbellata Mart. (MY.UMB), Gochnatia polymorpha (Less.) Cabrera (GO.POL) e Ocotea puberula (Rich.) Nees. (OC.PUB).

Revista Árvore, Viçosa-MG, v.38, n.3, p.469-478, 2014 
Tabela 2 - Índices de invasão biológica nos fragmentos e estratos estudados em Lages, SC, sendo estes calculados para as diferentes distâncias da borda e para a área total. AF1 = estrato adulto do fragmento no PARNAMUL, RF1 = estrato regenerante do fragmento no PARNAMUL, AF2 = estrato adulto do fragmento na EPAGRI e RF2 = estrato regenerante do fragmento na EPAGRI. $p$ = significância do coeficiente angular do ajuste linear entre IIB e a distância da borda.

Table 2 - Biological invasion index in the studied fragments and components in Lages, SC, calculated to different distances from the edge and total area. AF1 = adult component of PARNAMUL fragment, RF1 = regenerative component of PARNAMUL fragment, AF2 = adult component of EPAGRI fragment and RF2 = regenerative component of EPAGRI fragment. $p=$ angular coefficient significance of the linear adjustment between BII and the distance from the edge.

\begin{tabular}{lcccr}
\hline & AF1 & RF1 & AF2 & RF2 \\
\hline Borda & 0,09 & 0,23 & 0,49 & 0,99 \\
$\mathbf{2 0} \mathbf{~ m}$ & 0 & 0 & 0,85 & 1,32 \\
$\mathbf{4 0} \mathbf{~ m}$ & 0 & 0 & 0,32 & 1,00 \\
$\mathbf{6 0} \mathbf{~ m}$ & 0,08 & 0 & 0,24 & 1,27 \\
$\mathbf{8 0} \mathbf{m}$ & 0,06 & 0 & 0,36 & 0,69 \\
Total & 0,05 & 0,54 & 0,61 & 1,96 \\
$\boldsymbol{p}$ & 0,93 & 0,50 & 0,31 & 0,50 \\
\hline
\end{tabular}

No componente adulto do F2 (Figura 1B), Ligustrum lucidum (LI.LUC) agrupou-se com Lithraea brasiliensis Marchand (LI.BRA), Matayba elaeagnoides Radlk. (MA.ELA) e Zanthoxylum kleinii (R.S.Cowan) P.G.Waterman (ZA.KLE). No componente regenerativo do F2 (Figura 1C), Ligustrum sinescens (LI.SIN) ocorreu nos mesmos locais que Allophylus guaraniticus (A.St.-Hil.) Radlk. (AL.GUA), Calyptranthes concinna DC. (CA.CON) e Dicksonia sellowiana (DI.SEL); e Ligustrum lucidum aconteceu em Allophylus edulis (A.St.-Hil., Cambess. \& A.Juss.) Radlk. (AL.EDU), Myrcia palustris DC. (MY.PAL) e Banara tomentosa (BA.TOM). O componente regenerativo do $\mathrm{F} 1$ não possuiu espécies invasoras com cinco ou mais indivíduos.

\section{DISCUSSÃO}

Considerando-se ambos os fragmentos e componentes avaliados, o componente regenerante do F1 se destacou como o de menor riqueza, o que pode ser explicado pela presença abundante de taquara nesse fragmento, que, segundo Santos et al. (no prelo), pode inibir a regeneração natural. No entanto, em todos os casos houve elevada riqueza de Myrtaceae, corroborando outros estudos realizados na FOM do Planalto Sul-Catarinense, que indicam que essa é uma importante família na região (e. g. KLAUBERG et al., 2010; HIGUCHI et al., 2012a, b; SILVA et al., 2012; HIGUCHI et al., 2013). Casearia decandra, que se destacou em IVI, foi relatada por Klauberg et al. (2010) também como a espécie de maior importância relativa $(\mathrm{IVI}=6,69 \%)$.
$\mathrm{O}$ alto valor de IIB encontrado no F2, especialmente no estrato regenerante, indica a elevada capacidade invasora das espécies exóticas, principalmente de Ligustrum lucidum, espécie invasora de maior abundância na área. A expressiva participação dessa espécie ocorreu devido à proximidade do fragmento com o meio urbano, pois é a cultivada na arborização urbana, e à sua facilidade de dispersão por aves, indicando elevada capacidade regenerativa, o que sugere que essa espécie esteja bem adaptada ao ambiente. Aragón e Gloom (2003) destacaram a capacidade de L. lucidum em produzir grande quantidade de sementes e germinar e sobreviver em grande variação de ambientes florestais. O fato de espécies desse gênero já terem sido relatadas em outros estudos fitossociológicos nessa fitofisionomia (CORDEIRO; RODRIGUES, 2007) reforça o potencial invasor de Ligustrum spp. na região.

As espécies do gênero Pinus, principalmente Pinus taeda no F1, ocorreram devido à proximidade do fragmento com plantios dessas espécies, amplamente cultivada na região para fins madeireiros e de produção de celulose. Zanchetta e Diniz (2006) descreveram em seu estudo a facilidade de espécies do gênero Pinus em estabelecer-se em fragmentos florestais, especialmente locais com grande insolação, como bordas de matas.

Apesar de alguns autores já terem citado a relação entre a distância da borda e a ocorrência de espécies invasoras (BROTHERS; SPINGARN, 1992; HONNAY et al.; 2002), neste estudo não houve correlação linear. Isso provavelmente ocorreu porque a espécie invasora de maior abundância, L. lucidum, possui elevada 


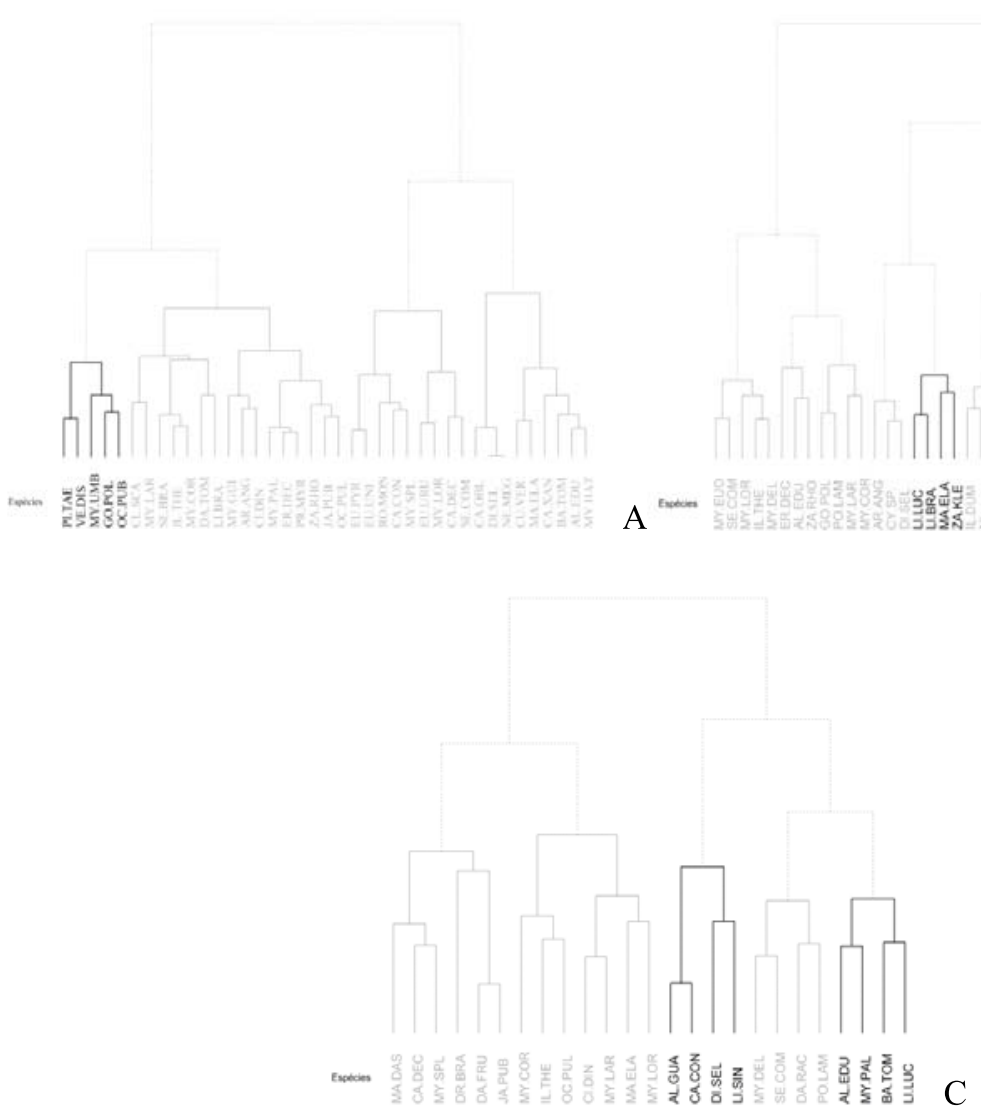

Figura 1 - Agrupamentos entre espécies arbóreas exóticas (PI.TAE = Pinus taeda; LI.LUC = Ligustrum lucidum; LI.SIN = Ligustrum sinescens) (abundância $\geq 5$ indivíduos) e nativas em fragmentos de Floresta Ombrófila Mista em Lages, SC. A = estrato adulto do PARNAMUL (F1), B = estrato adulto da EPAGRI (F2) e C = estrato regenerante da EPAGRI (F2).

Figure 1 - Grouping between exotic (PI.TAE = Pinus taeda LI.LUC = Ligustrum lucidum; LI.SIN = Ligustrum sinescens) (abundance $\geq 5$ individuals) and native tree species in Mixed Ombrophylous Forest fragments in Lages, SC. A $=$ PARNAMUL (F1) adult component, $B=E P A G R I(F 2)$ adult component and $C=E P A G R I(F 2)$ regenerative component.

plasticidade ecológica e é tolerante ao sombreamento, ocorrendo tanto na borda quanto no interior do fragmento. Esse padrão pode ser confirmado quando se observam as espécies associadas a L. lucidum, que pertencem a diferentes guildas de regeneração. Lithraea brasiliensis, por exemplo, é uma espécie inicial de sucessão que, segundo Scipioni et al. (2013), pode ser classificada como pioneira. Matayba elaeagnoides, Myrcia palustris e Allophylus edulis são classificadas, pelos referidos autores, como clímax exigente de luz, enquanto Banara tomentosa o é como clímax tolerante à sombra.

Revista Árvore, Viçosa-MG, v.38, n.3, p.469-478, 2014
Pinus taeda, que pode ser classificado como pioneiro, tendeu a ocupar o mesmo espaço de espécies iniciais de sucessão. Essa espécie se agrupou a Vernonanthura discolor (pioneira, segundo FERREIRA et al., 2013), Gochnatia polymorpha (pioneira, segundo SCIPIONI et al., 2013) e às clímax exigentes de luz Myrsine umbellata e Ocotea puberula (classificadas segundo SCIPIONI et al., 2013). Ligustrum sinescens, espécie de pequeno porte, tendeu a se agrupar com espécies típicas de sub-bosque, como Allophylus guaraniticus, Calyptranthes concinna e Dicksonia sellowiana. Esses agrupamentos sugerem que espécies exóticas estão 
ocupando o local de nativas na estrutura da floresta e em diferentes guildas de regeneração, podendo ocorrer, ao longo do tempo, um processo de substituição de espécies.

\section{CONCLUSÃO}

O padrão de invasão biológica por espécies arbóreas nos fragmentos diferiu em função da influência da matriz de contato e da natureza ecológica das espécies invasoras, e não foi observada relação linear entre os valores de IIB e a distância da borda. Tal resultado pode ser explicado pelas características ecológicas das principais espécies invasoras (Pinus taeda e Ligustrum lucidum) e pelo estágio sucessional em que se encontram os fragmentos, considerado avançado. No F1, a invasão ocorreu com baixa representatividade em número de indivíduos, com destaque para Pinus taeda, que se agrupou com espécies pioneiras, o que indica o potencial dessa espécie de substituir espécies dessa guilda de regeneração e de ser colonizadora de áreas com maior luminosidade em ecossistemas florestais, como bordas, clareiras e áreas em início de estágio de sucessão. No F2, Ligustrum lucidum se destacou com elevada importância, tanto no componente adulto quanto no regenerativo, demonstrando a sua capacidade de estabelecimento e desenvolvimento. L. lucidum agrupou-se com espécies de diferentes guildas de regeneração, sugerindo seu grande potencial invasor em áreas de FOM.

\section{REFERÊNCIAS}

APG III. An update of the Angiosperm Phylogeny Group classification for the orders and families of flowering plants: APG III. Botanical Journal of the Linnean Society, v.161, n.2, p.105121, 2009.

ARAGÓN, R.; GROOM, M. Invasion by Ligustrum lucidum (Oleaceae) in NW Argentina: early stage characteristics in different habitat types.

Revista de Biologia Tropical, v.51, n.1, p.59-70, 2003.

BARBIERI, R. L.; STUMPT, E. R. T. Origem e evolução de plantas cultivadas. Brasília: Embrapa, 2008. 909p.

BOCARD, D.; GILLET, F.; LEGENDRE, P. Numerical Ecology with R. New York: Springer, 2011. 306p.
BROTHERS, T. S.; SPINGARN, A. Forest fragmentation and alien plant invasion of Central Indiana Old-Growth Forests. Conservation Biology, v.6, n.1, p.91-100, 1992.

CORDEIRO, J.; RODRIGUES, W. A. Caracterização fitossociológica de um remanescente de Floresta Ombrófila Mista em Guarapuava, PR. Revista Árvore, v.31, n.3, p.545-554, 2007.

FERREIRA, P. I. et al. Espécies potenciais para recuperação de áreas de preservação permanente no Planalto Catarinense. Floresta e Ambiente, v.20, n.2, p.173-182, 2013.

HELENO, R. et al. Evaluation of restoration effectiveness: community response to the removal of alien plants. Ecological Applications, v.20, n.5, p.1191-1203, 2010.

HIGUCHI, P. et al. Floristic composition and phytogeography of the tree component of Araucaria Forest fragments in southern Brazil. Revista Brasileira de Botânica, v.35, n.2, p.145-157, 2012a.

HIGUCHI, P. et al. Influência de variáveis ambientais sobre o padrão estrutural e florístico do componente arbóreo, em um fragmento de Floresta Ombrófila Mista Montana em Lages, SC. Ciência Florestal, v.22, n.1, p.79-90, 2012 b.

HIGUCHI, P. et al. Florística e estrutura do componente arbóreo e análise ambiental de um fragmento de Floresta Ombrófila Mista AltoMontana no município de Painel, SC. Ciência Florestal, v.23, n.1, p.153-164, 2013.

HONNAY, O.; VERHEYEN, K.; HERMY, M. Permeability of ancient forest edges for weedy plant species invasion. Forest Ecology and Management, v.161, n.1-3, p.109-122, 2002.

INSTITUTO BRASILEIRO DE GEOGRAFIA E ESTATÍSTICA - IBGE. Manual técnico da vegetação brasileira. Rio de Janeiro: 2012. 271p.

INSTITUTO HÓRUS. Instituto Hórus de desenvolvimento e conservação ambiental. Disponível em: <http:// www.institutohorus.org.br/> Acesso em: 17 jul. de 2013.

Revista Árvore, Viçosa-MG, v.38, n.3, p.469-478, 2014 
KLAUBERG, C. et al. Florística e estrutura de um fragmento de Floresta Ombrófila Mista no Planalto Catarinense. Biotemas, v.23, n.1, p.35-47, 2010.

LAURANCE, W. F. et al. Ecosytem decay of amazonian forest fragments: a 22-year investigation. Conservation Biology, v.16, n.3, p.605-618, 2002.

LEGENDRE, P.; BLANCHER, F. G. Species association. Edmonton: University of Alberta, 2008. 11p.

MULLER-DOMBOIS, D.; ELLENBERG, H. Aims and of vegetation ecology. New York: John Wiley \& Sons, 1974. 547p.

OKSAnEN, J. Cluster Analysis: tutorial with R. 12p. 2012. Disponível em: <http://cc.oulu.fi/ $\sim$ jarioksa/opetus/metodi/sessio3.pdf $>$ Acesso em: 3 ago. 2013.

OKSANEN, J. et al. Vegan: community ecology package. R package version 2.0-7. Disponível em: <http://CRAN.R-project.org/package=vegan> Acesso em: 3 ago. de 2013.

R DEVELOPMENT CORE TEAM. R: A language and environment for statistical computing. R Foundation for Statistical Computing, Vienna. Disponível em: <http://www.Rproject.org > Acesso em: 18 jul. de 2013.

SANTOS, K. F. et al. Regeneração natural do componente arbóreo após a mortalidade de um maciço de taquara em um fragmento de Floresta Ombrófila Mista em Lages, SC. Ciência Florestal, no prelo.
SCIPIONI, M. C.; GALVÃO, F.; LONGHI, S. J. Composição florística e estratégias de dispersão e regeneração de grupos florísticos em Florestas Estacionais Deciduais no Rio Grande do Sul. Floresta, v.43, n.2, p.241254, 2013.

SILVA, A. C. et al. Relações florísticas e fitossociologia de uma Floresta Ombrófila Mista Montana Secundária em Lages, Santa Catarina. Ciência Florestal, v.22, n.1, p.193-206, 2012.

VALÉRY, L. et al. In search of a real definition of the biological invasion phenomenon itself.

Biological Invasions, v.10, n.8, p.1345-1351, 2008.

VOLPATO, M. M. L. Regeneração natural

em uma floresta secundária no domínio de Mata Atlântica: uma análise fitossociológica. 1994. $123 f$. Dissertação (Mestrado em Ciência Florestal) Universidade Federal de Viçosa, Viçosa, MG, 1994.

ZANCHETTA, D.; DINIZ, F. V. Estudo da contaminação biológica por Pinus spp. em três diferentes áreas na Estação Ecológica de Itirapina (SP, Brasil). Revista do Instituto

Florestal, v.18, n.1, p.1-14, 2006.

ZILLER, S. R. Instituto para o

Desenvolvimento de Energias Alternativas e da Autosustentabilidade (Ideaas) PR. 2000. Disponível em: < http://www.ideaas.org > Acesso em: 17 jul. 2013. 